\title{
Characterization of Complete Denture: A Review
}

\author{
Dr. Ambuj Chandna, Dr. Abu Nedal, Dr. Tanvi Kalra, Dr. Yashpal Rana, \\ Dr. Ajay Yadav, Dr. Akash Jindal \\ Senior Lecturer At Divya Jyoti College Of Dental Sciences \&Amp; Research, Modinagar
}

\begin{abstract}
Despite the fact that solutions to functional and comfort problems are often available, successfully restoring the appearance of an edentulous patient remains a challenge. Esthetics pertains to the sense of the beautiful or the science which deduces from nature and taste the rules and principles of art. Esthetics has always exerted a profound influence in history. Because it has always been a prime concern of individuals, having the effect of exciting emotions of approval or disapproval.Significant improvements in facial esthetics and more rapid social integration of the patient is seen with use of characterized denture compared to conventional denture.
\end{abstract}

Keywords: Characterization, prosthesis, conventional denture prosthesis, denture esthetics.

\section{Introduction}

The glossary of prosthodontic term defines esthetics is the branch of philosophy dealing with beauty, especially with the components there of, viz., color and form; and the qualities involved in the appearance of a given restoration. Treatment challenges for completely edentulous patient have traditionally been described as a combination of esthetics, function and comfort. Some studies regarding predictors of complete denture success failed to show esthetics as more important than function or comfort. In contrast to these studies, Carlsson et al found that esthetics was the factor most responsible for complete denture success.

A survey by Vallittu et al found that patients wearing removable dentures considered the appearance to be the most important property of the teeth.

According to the glossary of prosthodontics terms "Denture characterization is modification of the form and color of the denture base and teeth to produce a more lifelike appearance." As said by Frush and Fisher, "the environment of the teeth is as important as the tooth itself". Thus the two elements that must be considered in denture esthetics are teeth and their supporting denture base. Complete dentures must be esthetic as well as functional.Hardy stated that, "To meet the esthetic needs of the denture patient, we should make the (denture) teeth look like (the patient's) natural teeth."

\section{Methods of characterization:}

Complete denture can be characterized by two basic methods.

1. Characterization by selection, arrangement and modification of artificial teeth.

2. Characterization by tinting the denture bases.

\section{Characterization By Selection: (Arrangement And Modification Of Artificial Teeth)}

The teeth can be modified to harmonize with the patient's age, sex, and personality to provide subjective unity. Fisher said that gender, personality, and age can be used as guidelines for tooth selection, arrangement, and characterization to "enhance the natural appearance of the individual. The ways of characterization are;

1. Varying the direction of the long axis of teeth.

2. Place the teeth so that the tips of the maxillary lateral incisors show when the patient speaks seriously; the amount depends on the age and sex, less for old than for young people and more for woman than for men.

3. Create asymmetry in the divergences of the proximal surfaces of the teeth from the contact points. Martone stated that, "The key to esthetics lies in asymmetry."Most things in nature are asymmetric, and in the human face many minute and subtle differences are found from one side to the other.

4. Use an eccentric midline.

5. Place one maxillary central and lateral incisor parallel to the midline and rotate the other central and lateral incisors slightly in a posterior direction.

6. Place one maxillary central incisor slightly in an anterior direction to the other central incisor.

7. Place the neck of one maxillary central incisor in a posterior direction and the neck of other central incisor in an anterior direction.

8. Create asymmetry for the maxillary right and left cuspids. Rotate one in a posterior direction than the other. 
9. Gingival tissues recede with age. Selecting a long tooth, contouring the wax to show gingival recession and then staining it a bit, can give natural appearance, can reproduce this recession. Long clinical crowns with receded gingiva after periodontal destruction, may also play a role in achieving a natural-looking denture for selected patients.

10. Grinding the incisal edges. Teeth abrade with age. Reshaping the incisal edges and mesiodistal diameter makes it possible to modify any tooth to the desired form.

11. A teeth arrangement that is too perfect may not be ideal. In fact, slight modifications in the position of teeth such as overlapping, tilting, rotation and incisal variations may contribute to a natural-looking denture.

12. Spacing and diastemas often exist in natural dentition. Thus slight diastema can be created between the lateral incisor and the cuspid on one side. The wearing away of the natural teeth at the contact points creates spaces between the teeth. The migration of teeth also creates spaces. To simulate the wear by positioning the artificial teeth to create spaces, can give a natural appearance. Diastema given should exceed $2-3 \mathrm{~mm}$ and should be wider at the incisal edge than the base. In diastemas smaller than 2-3 mm, fibrous food tends to be trapped and can be a source of embarrassment.

13. A hair line crack can be given in the teeth.

14. Often, gold or alloy restorations can be placed in these teeth to create the illusion of naturalness. The use of gold occlusal surfaces on the teeth of prosthesis can contribute to its clinical success.

15. Silver filling can be given on posterior teeth.

16. Cast crown can be given on posterior teeth. Some patients who seek new dentures ask that a metal crown be placed in the denture to resemble their natural dentition.

17. A discolored tooth (as R.C.T treated) can be shown by selecting one or two teeth of darker shade compared to the rest of the teeth set. Older patients tend to have darker teeth as a result of discoloration from fillings and food stains.

Stock artificial teeth as provided by manufacturers have a uniformity of color and shade, inherent to their production that does not impart a natural esthetic result to dentures. Methods have been developed for making acrylic resin teeth by incorporating staining and characterization to natural denture teeth.

\section{Characterization Of The Denture Bases}

Pound in 1951 incorporated the racial and individual colour peculiarities, of the gingiva in artificial denture. He was the first to suggest a method of tinting acrylic denture bases to simulate the gingival colour. Kemnitzer used a combination of blue and brown stain to reproduce the melanotic pigmentation of the gingiva.

\section{Indication for characterization of denture base \\ 1. Patients with an active upper lip. \\ 2. Patients with a prominent pre-maxillary process. \\ 3. Actors, singers and others who may expose gum tissues areas during their performances. \\ 4. The psychological acceptance of the dentures by the patient.}

The smooth, pink, polished surface of an average acrylic denture quickly reveals it's false. Therefore, the surface of the denture base can be modified by

Stippling: Lynn C. Dirksen described a procedure which provides an inexpensive means of obtaining more natural appearing buccal and labial contours for complete dentures. The stippled surface produced by these plastic veneer forms enhances the esthetic appearance of plain pink acrylic resin. The ultimate esthetic result, however, is obtained by the combination of stippling and tinting. The stippled plastic contour veneer is applied to a wax-up in approximately five minutes, and the carving and polishing of the buccal and the labial surfaces of the cured denture is practically eliminated.Suresh Nayar and Nicholas W. Craik had stated that gingival stippling is a characteristic of the healthy attached gingiva. Copying gingival texture and contours contributes to the natural appearance of labial flanges in complete dentures by causing uneven reflection of light. This, in turn, reduces the shine and reflection typically seen in highly polished denture flanges and provides a more natural appearance.

Alveolar eminence: To further enhance the natural effect, it is necessary to imitate the anatomy the gingivae and alveolus. The labial flange of a complete denture should not be a smooth curved sheet of acrylic, but instead should show a series of swellings corresponding to the alveolar eminencies over the roots of the teeth. These are most marked anteriorly and become progressively less marked in the pre-molar and molar region. In the upper anterior region, the canine eminence is most marked. The lateral incisor eminence is small. In the lower jaw, again the canine eminence is most marked and a series of smaller ridges mark the presence of the incisor roots. 
Inflamed or bulbous gingiva: The reflection of inflamed or bulbous gingiva can also be reproduced by leaving more interdental wax.

Use of tints in the denture bases: Several methods have been used to tint denture, base resins to achieve a more natural appearance. Usually heat curing or auto-polymerizing resins of various shades or colors are painted on the denture base or are shifted on to the mold during denture construction to obtain a tinted denture. Most widely used tints today are the various pigments, which are placed with in the original mold chamber, so they do not affect contours

\section{Ideal requirements of denture base tinting materials}

1. It should be readily miscible with methyl methacrylate resin.

2. It should be non-toxic

3. It should not add appreciable bulk to denture bases.

4. It should be stable and non-fading.

5. It should be resistant to loss from abrasion in cleaning and in normal function.

6. It should not alter the properties of the denture base resins.

Additional shades aside from the standard Kayon Tints can be mixed from earth color pigments which enable the dentist to match virtually any color of gingival tissue. Most widely used tints are the Kayon dental stains or tinting resins. One Kayon Kit contains five shades.

\section{Conclusion}

The treatment with characterized complete denture prosthesis can produce a more lifelike ornatural appearance compared with conventionaldenture that produces artificial look in edentulous patients. Prosthodontic therapy with use of characterized complete denture prosthesis compare to conventional denture prosthesis aids the patient in developing proper speech, enhanced esthetics and may have dramatic social and psychological benefits for these patients.

\section{Refrences}

[1]. 1.Yoshizumi DT. An evaluation of factors pertinent to the success of complete denture service.J Prosthet Dent 1964; 14:866-78.

[2]. Carlsson GE, Otterland A, Wennstrom A, Odont D. Patient factors in appreciation of complete dentures. J Prosthet Dent 1967; 17:322-8.

[3]. Vig RG. The denture look. J Prosthet Dent 1961:9-15

[4]. Vallittu PK, Vallittu ASJ, Lassila VP. Dental aesthetics -a survey of attitudes in different groups of patients. J Dent 1996; 24:335-8.

[5]. Ruel-Kellermann M. What are the psychological factors involved in motivating individuals to retain their teeth? Dreams and facts. Int Dent J 1984;34:105-109.

[6]. Frush JP, Fisher RD. Introduction to dentogenic restorations. J Prosthet Dent 1955; 5:586-95.

[7]. Waliszewski M. Restoring dentate appearance: A literature review for modern complete denture esthetics

[8]. J Prosthet Dent 2005; 93:386-94.

[9]. Hirsch B, Levin B, Tiber N. Effects of patient involvement and esthetic preference on denture acceptance. J

[10]. Prosthet Dent 1972; 28:127-32.

[11]. 9. Spear FM, Kokich VG. A Multidisciplinary Approach to Esthetic Dentistry. Dent Clin North Am 2007; 51:487-505 